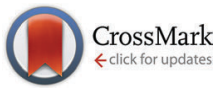

Cite this: Phys. Chem. Chem. Phys., 2017, 19, 3627

Received 1st November 2016, Accepted 17th December 2016

DOI: $10.1039 / c 6 c p 07485 e$

www.rsc.org/pccp

\section{Impact of morphology on polaron delocalization in a semicrystalline conjugated polymer $\dagger$}

\author{
Robert Steyrleuthner, ${ }^{\star a}$ Yuexing Zhang, ${ }^{\text {bc }}$ Lei Zhang, ${ }^{d}$ Felix Kraffert, ${ }^{a}$ \\ Benjamin P. Cherniawski, ${ }^{d}$ Robert Bittl, ${ }^{a}$ Alejandro L. Briseno, ${ }^{d}$ Jean-Luc Bredas ${ }^{b}$ \\ and Jan Behrends*a
}

\begin{abstract}
We investigate the delocalization of holes in the semicrystalline conjugated polymer poly(2,5-bis(3alkylthiophene-2-yl)thieno[3,2-b]thiophene) (PBTTT) by directly measuring the hyperfine coupling between photogenerated polarons and bound nuclear spins using electron nuclear double resonance spectroscopy. An extrapolation of the corresponding oligomer spectra reveals that charges tend to delocalize over 4.0-4.8 $\mathrm{nm}$ with delocalization strongly dependent on molecular order and crystallinity of the PBTTT polymer thin films. Density functional theory calculations of hyperfine couplings confirm that long-range corrected functionals appropriately describe the change in coupling strength with increasing oligomer size and agree well with the experimentally measured polymer limit. Our discussion presents general guidelines illustrating the various pitfalls and opportunities when deducing polaron localization lengths from hyperfine coupling spectra of conjugated polymers.
\end{abstract}

\section{Introduction}

Organic semiconductors based on conjugated polymers represent a fascinating class of materials not only due to their multiple applications in functional devices but also their rich fundamental chemistry and physics. Charge carriers in conjugated polymers do not generally move freely in electronic bands, but self-localize in the form of polarons due to the significant coupling between the electronic and geometric structures (strong electron-phonon couplings). ${ }^{1}$ Thus, the lattice around an excess charge relaxes via bond length or torsional angle deformations, giving rise to the binding energy of the polaron. This implies that even in a completely planar or rigid conjugated chain without any defects, the polaron wave function is limited to a certain intrinsic/effective molecular length. However, most conjugated polymers exhibit a high conformational degree of freedom along the chains leading to partial breaks in conjugation. If the average length of coherent molecular subunits ( $\pi$-systems) is shorter than the intrinsic extension of the polaron, the delocalization becomes effectively confined by the morphology of the system. As precise knowledge of polaron

\footnotetext{
${ }^{a}$ Freie Universität Berlin, Berlin Joint EPR Lab, Institut für Experimentalphysik, Berlin, Germany. E-mail: robert.steyrleuthner@fu-berlin.de, j.behrends@fu-berlin.de

${ }^{b}$ King Abdullah University of Science \& Technology, Solar \& Photovoltaics Engineering Research Center, Thuwal 23955-6900, Saudi Arabia

${ }^{c}$ Department of Chemistry, Hubei University, Wuhan 430062, China

${ }^{d}$ Department of Polymer Science and Engineering, Conte Research Center, University of Massachusetts, 120 Governors Drive, Amherst, MA, 01003, USA

$\dagger$ Electronic supplementary information (ESI) available. See DOI: 10.1039/c6cp07485e
}

extension in conjugated polymers remains rare, the nature of the limiting process for a particular material also remains unclear.

The lack of exact, especially experimental information on polaron delocalization contrasts with the significant influence that localization of charge carriers has on the fundamental physico-chemical processes in organic semiconductors. Charge transport is usually described as a hopping process among localized states that are distributed in energy. ${ }^{2-4}$ In this framework the carrier mobility mainly depends on the width of the (Gaussian) distribution. In disordered or completely amorphous semiconductors, charges can get trapped in the tail of the broad density of states (DOS), which reduces the carrier mobility drastically. On the other hand, if the DOS is narrowed (e.g., by local ordering or crystallization of the polymer), charge transport can get dominated by the molecular reorganization accompanied with the hopping process between adjacent, nearly isoenergetic states (polaronic transport). ${ }^{5}$ Beyond those two well established concepts, Hoffman et al. recently highlighted the importance of charge carrier delocalization in order to explain the difference in mobility in a series of related conjugated polymers exhibiting similar energetic disorder and reorganization energies. ${ }^{6}$ Furthermore, very high mobilities on the order of, or over $1 \mathrm{~cm}^{2} \mathrm{~V}^{-1} \mathrm{~s}^{-1}$ have been reported in recently developed conjugated polymers and are attributed to either preferential chain orientation or polymer structures that are resilient to torsions (i.e. by chemical bridging of aromatic units), therefore decreasing the energetic disorder and at the same time extending the delocalization of the $\pi$-electron system along the backbone. ${ }^{7-17}$ The influence of extended delocalization is corroborated by 
studies where experimental in-plane alignment of polymer backbones lead to a further substantial mobility increase in this particular transport direction. ${ }^{18-20}$ In addition to the importance of polaron delocalization for efficient transport of charges in organic semiconductors, there are several experimental results and theoretical models suggesting that extended delocalized states take part in the ultra-fast charge separation process at the donor-acceptor interface in organic solar cells. ${ }^{21-26}$ As both charge separation and transport are fundamental processes in functional devices, deeper insight into the delocalization of charges would significantly improve the understanding of the operation of organic solar cells, transistors, and light-emitting diodes.

A material that has been intensively studied in the past years with respect to fundamental correlations between molecular structure and charge carrier transport and to applications in organic solar cells, is the conjugated polymer poly(2,5-bis(3alkylthiophene-2-yl)thieno[3,2-b]thiophene) (PBTTT) (Fig. 1). Its characteristic semicrystalline morphology in thin polymer layers comprising of $\pi$-stacked polymer chain lamellae has been put forward as one explanation for the high hole mobility of up to $1 \mathrm{~cm}^{2} \mathrm{~V}^{-1} \mathrm{~s}^{-1}$ measured in field-effect transistors. ${ }^{27-38}$ It was also proposed from theoretical modeling that a low density of trap states and high delocalization of charges along the planarized chains in closely packed lamellar structures is responsible for the improved mobility in PBTTT. ${ }^{39}$ It is therefore highly desirable to have a direct measure of the polaron (de)localization in PBTTT in order to correlate with the structure and transport properties of the polymer.

While a wealth of studies focused on a structural analysis in order to explain the high charge carrier mobility in PBTTT, the fundamental phenomenon of charge delocalization along the backbone has to date only been accessed theoretically. ${ }^{39,40}$ Very recently, we reported the synthesis of BTTT oligomers to shed light on the evolution of crystalline behavior, morphology, and interaction with the fullerene acceptor as the molecular conjugation length increases. ${ }^{41}$ In turn, this series of compounds now enables us to investigate directly the delocalization of charges in PBTTT in a systematic way via electron nuclear double resonance (ENDOR) spectroscopy, without having to rely exclusively on advanced modeling. In conjugated polymers, the length of the polymer chain can severely influence factors such as solubility, film formation, crystallinity, and consequently charge transport or carrier generation in functional devices. ${ }^{42}$ By investigating two molecular weight fractions of PBTTT $\left(M_{\mathrm{n}}=15 \mathrm{kDa}\right.$ and $20 \mathrm{kDa}$ ), we illustrate a case where two chemically similar polymers show a different degree of polaron localization. In combination with complementary quantum chemical calculations of the well-defined oligomer molecules, we have been able to gain deeper insight into the factors that limit delocalization along the polymer chain and how delocalization depends on structure in thin films. In addition, our discussion presents a general guideline illustrating the pitfalls and opportunities when trying to deduce polaron localization lengths from hyperfine coupling spectra of conjugated polymers.

\section{ENDOR spectroscopy to determine charge localization}

An early attempt to reveal the localization of polarons in several conjugated polymers made use of a correlation between the energy of photo- or doping-induced polaron absorption ${ }^{43-46}$ and the effective conjugation length, ${ }^{47}$ stimulating further investigations. $^{48-51}$ Here, we apply an alternative technique based on electron paramagnetic resonance (EPR), which has proven to be very successful in determining the extension of excited species, especially in bio-related molecules/systems. ${ }^{52-54}$ In general, EPR is capable to identify unpaired electrons due to the Zeeman splitting under an applied magnetic field by microwave spin manipulation; in this context, it has been demonstrated to reliably detect photo-generated polarons in different types of organic solar cells. ${ }^{55,56}$ The interaction between the magnetic dipole of a nucleus (with nonzero spin), bound to the chemical structure of the material, and the spin of a charge carrier leads to the formation of additional hyperfine sublevels, which increases the number of EPR transitions. In case of a hydrogen nucleus $(I=1 / 2)$ in the vicinity of an unpaired electron $(S=1 / 2)$, the hyperfine interaction leads to an additional splitting of the spin up and the spin down state, respectively, resulting in two EPR and two NMR transitions (see Fig. 2). The magnitude of the energetic splitting $a$ (hyperfine splitting constant) depends on the strength of the dipole-dipole interaction and can be

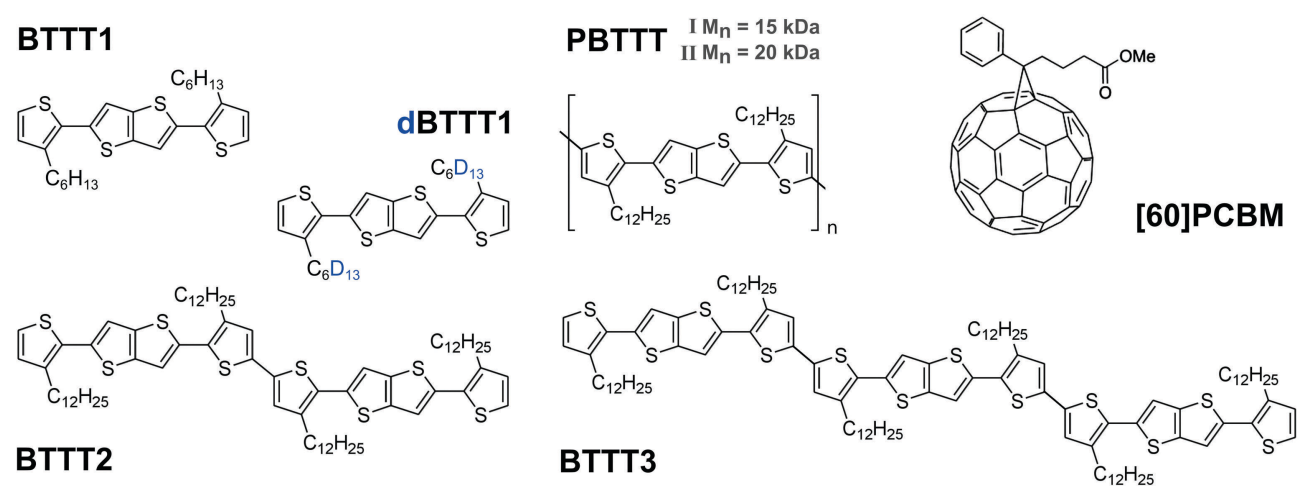

Fig. 1 Chemical structures of the BTTT oligomers/polymers blended at 1:1 weight ratio with a fullerene acceptor. 


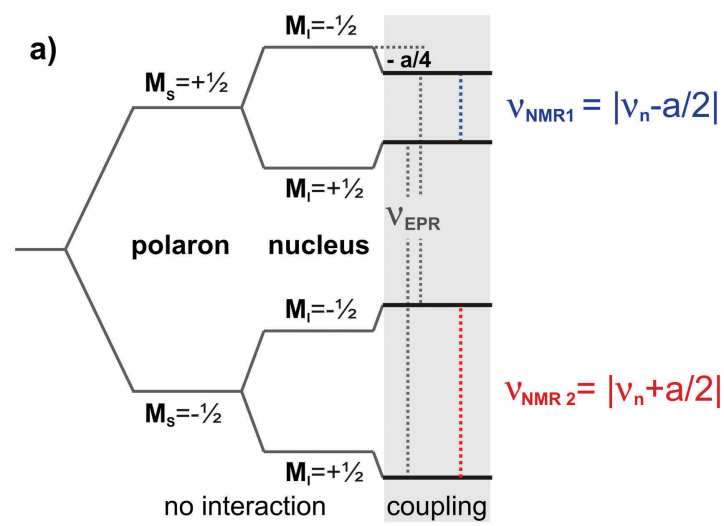

b)

spectrum showing the resonance deviation from the free nucleus frequency in the case of hyperfine interaction with a polaron.

divided into two contributions. The isotropic contribution to the hyperfine coupling (HFC) is based on the polaron wave function probability amplitude at the position of the nucleus given by the Fermi contact term. The second part is anisotropic in nature and related to distance and relative orientation of polaron spin and nucleus spin. As the HFC strength is proportional to the spin/charge carrier density at the location of the nucleus, measuring $a$ is therefore an efficient tool to determine the spatial extent of polaron wave functions.

The hyperfine coupling strength can directly be resolved by ENDOR spectroscopy. This technique exploits the influence of resonant radiofrequency (RF) pulses (exciting NMR transitions) on EPR transitions (see Fig. 2a) with enhanced sensitivity compared to NMR. ${ }^{57}$ For a discrete isotropic coupling of a polaron spin to a single nucleus an exemplary ENDOR spectrum shows resonances at $-a / 2$ and $+a / 2$ centered around the free nuclear Larmor frequency $\nu_{\mathrm{n}}$ (see Fig. 2b). Therefore, stronger couplings result in broader ENDOR spectra indicating a high degree of polaron localization.

Several earlier studies have applied spin resonant techniques in order to determine the spatial extent of charge carriers in organic semiconductors. Polarons in polyphenylenevinylene, polythiophene or polyfluorene derivatives were either generated by intrinsic defects, chemical doping, or by light excitation in mixtures with e.g. a fullerene acceptor. ${ }^{58-64}$ Very recently, the electronic structure of positive polarons in a small conjugated molecule for application in solar cells or OFETs was investigated by resolving the proton hyperfine spectrum. ${ }^{65}$ The technique was also applied to study low band-gap polymers and investigate correlations between the charge separation efficiency and polaron delocalization in solar cells. ${ }^{66}$ However, one major drawback of using hyperfine spectra in order to assess charge delocalization in organic semiconductors is the complexity of the analysis. It is most convenient to investigate the coupling between the polaron spin and the nuclear spin of hydrogen protons as the natural abundance of carbon isotopes exhibiting nuclear spin is just around $1.07 \%$ (versus $99.98 \%$ for ${ }^{1} \mathrm{H}$ ). The spatial extent of excitations such as excitons or polarons covers typically several repeat units of the polymer chain. Consequently, the charge carrier spin is coupled to a number of protons bound to the aromatic rings and protons attached to aliphatic side chains, which prevents the resolution of single proton couplings in conjugated polymer films. Additional broadening of single resonances occurs because the anisotropic portion of the HFC in thin polymer films is not reduced by motional averaging, as usually seen for liquid samples. This underlines the need for simulating the spectra as a sum of contributions from several nuclei partially overlapping in their spectral appearance. In order to do so, the HFCs in a series of ionized oligomers with increasing degree of polymerization have been calculated via DFT (B3LYP functional) and directly compared with the experimental spectrum of the corresponding polymer to assess the localization length. ${ }^{66}$ While this is indeed a practical procedure, the final result is in fact severely influenced by the level of theory and structural model chosen for calculations making this comparison alone arguable. Particularly, recent theoretical work has shown that modern long-range corrected density functionals can minimize electron self-interaction errors and provide the right balance between localization and delocalization effects. ${ }^{67,68}$ This was confirmed in one lately published computational study of polaron delocalization in PBTTT oligomers. ${ }^{40}$

Additional uncertainty comes from the fact that exact information on the conformation of the polymer is missing and the solubilizing side chains containing a large number of hydrogen nuclei are usually not considered or truncated in quantum chemical calculations. We will show here that a reliable simulation requires additional knowledge on the structural conformation of the polymer including its side chains.

An approach to eliminate to some extent these uncertainties was performed in 1991 already by Brendel $e t$ al. who measured the hyperfine coupling in a series of n-type doped phenylenevinylene oligomers in frozen solution. ${ }^{59}$ As expected, the width of the ENDOR spectrum narrows with increasing oligomer length, corresponding to weaker hyperfine coupling and enhanced delocalization of the negative polaron. This effect is demonstrated even more clearly in a recent publication by Wilson et al. who determined the HFCs of perylenediimide (PDI) oligomers designed for conjugated molecular wires. ${ }^{69}$ The HFC of spectrally resolved protons exactly halved when doubling the molecule size and shows nearly a third of the 
monomer values for the trimer. Having an oligomer series at hand corresponding to the conjugated polymer under investigation, therefore gives the possibility to determine the spatial extent of polarons by observing the saturation of HFC independently of any model. Additionally, it represents an ideal test bed for quantum chemical models on experimentally existing oligomers with defined degree of polymerization. We note that this oligomer approach is also very common to assess the conjugation length (polymer limit) of a particular polymer from optical gap measurements as a function of oligomer length, reviewed by Gierschner et $a .^{70}$ Because PBTTT has shown to be applicable as donor material in organic solar cells, we will investigate the delocalization of holes after dissociation of light-generated excitons at the polymer/fullerene interface. Doing so also insures that the polarons characterized here have the same physical nature as the charges formed in functional devices, which is not necessarily the case for e.g. alkali metal doped polymers previously investigated by ENDOR spectroscopy.

\section{Results}

The EPR spectra of light-induced polarons generated in the BTTT oligomer/polymer:[60]PCBM mixtures are presented in the ESI $\dagger$ (Fig. S1). The spectra directly show that separated charges are generated for all material mixtures in the donor and acceptor phases after light excitation. The spectral signatures of the positive polaron located on the oligomer/polymer and the electron in PCBM are well separated at Q-band (34 GHz microwave frequency), which allows us to address the HFC of each component individually by choosing the magnetic field appropriately. The EPR signal intensity or charge generation efficiency decreases continuously when going from the polymer to the smallest BTTT1 oligomer; this is due to the energy mismatch for separation in accordance with previous results on BTTT oligomer solar cells. ${ }^{41}$ A detailed discussion of the spectra and a decomposition of the various influences that may change the spectral shape is, however, beyond the scope of this work; rather, we focus here on the investigation of the HFC.

\section{Oligomer/polymer ENDOR overview}

Fig. 3a shows the ${ }^{1} \mathrm{H}$-Davies ENDOR spectra of BTTT oligomer/ polymer:[60]PCBM mixtures measured at the spectral position of the positive polaron. All spectra were shifted by the resonance frequency of a free hydrogen nucleus $\nu_{\mathrm{H}}$ (at Q-band approx. $53 \mathrm{MHz}$ ). To increase the signal to noise ratio, the dBTTT1/ BTTT1 spectra were symmetrized by mirroring at $\nu_{\mathrm{H}}$. The general trend of decreasing hyperfine coupling strength with increasing molecular length appears clearly and points to an extended delocalization of the polaron wavefunction in larger molecules. In addition, the spectral shape is independent of temperature between $10 \mathrm{~K}$ and $80 \mathrm{~K}$ (see the example of PBTTT in the ESI, $\dagger$ Fig. S2), which implies that polarons do not hop incoherently between states within the time scale of the experiment and we directly probe the coherent extension of the excitation. Without any further sophisticated analysis, we extract the maximum HFC as interpolated from the edge of the ENDOR spectrum and plot it over the inverse number of BTTT units $(1 / N)$ in Fig. 3c. The maximum HFC exactly halves when going from BTTT1 to BTTT2 and is a third of the original value for BTTT3, as illustrated by the linear dependence presented over $1 / N$. This behavior suggests that the polaron extension spreads rather continuously along the polymer backbone and shows no or little saturation in this molecular length scale regime. We like to point out that several reports on conjugated polymers also indicate a spread of the polaron wave function in two dimensions to neighboring molecules or chains. ${ }^{45,46,71}$ This interchain effect would lead to an additional narrowing of the individual ENDOR spectra as the polaron effectively experiences weaker couplings. Although we can not exclude this effect for PBTTT completely, the continuous trend in Fig. 3c is a strong indication that for this system delocalization evolves predominantly along the conjugated backbone.
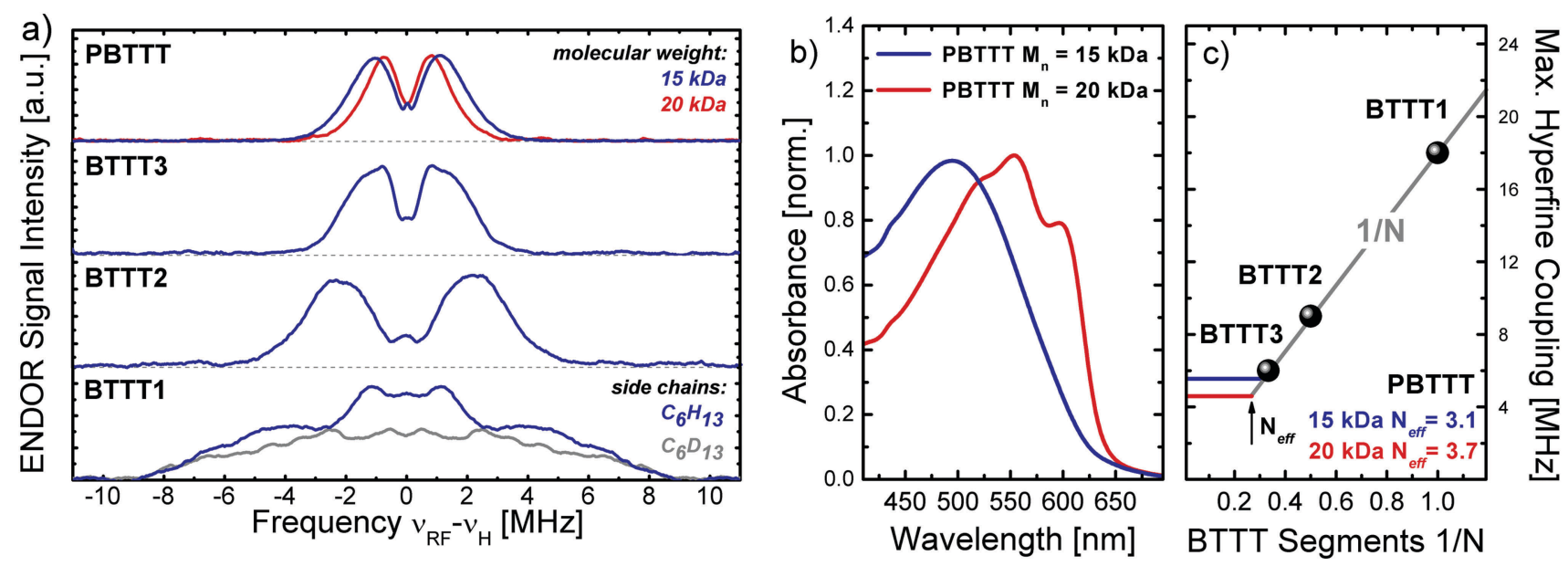

Fig. 3 (a) ${ }^{1} \mathrm{H}$-Davies ENDOR spectra of BTTT oligomers and PBTTT in mixture with [60]PCBM measured at the magnetic field position of the positive polaron (128 ns inversion pulse, $10 \mathrm{~K}$ ). Also shown is the ${ }^{1} \mathrm{H}$-Davies ENDOR spectrum for the side chain deuterated compound dBTTT1 in gray. (b) UV-vis absorption of thin PBTTT:[60]PCBM films. (c) Maximum experimental hyperfine coupling extracted from the edge of the HFC spectra as a function of oligomer length. $N_{\text {eff }}$ visualizes the effective positive polaron delocalization length for the two PBTTT fractions. 
Interestingly, the two molecular weight $\left(M_{\mathrm{n}}\right)$ fractions of PBTTT present remarkable differences in their hyperfine coupling spectra. By extrapolating the effective localization length $N_{\text {eff }}$, it can be concluded that the lower $M_{\mathrm{n}}$ fraction of PBTTT shows a localization length of $c a .4 \mathrm{~nm}$, corresponding to 3.1 BTTT units, i.e. almost identical to BTTT3, although the chain is approx. 7 times longer than this particular oligomer. However, delocalization in the PBTTT with the higher molecular weight extends significantly farther to $4.8 \mathrm{~nm}$, corresponding to 3.7 BTTT units.

The different degree of delocalization in the two chemically equivalent polymers can be explained by the influence of molecular weight on the structure formation and ordering of the films. To gain deeper insight into this process, we measured the UV-vis absorption of thin PBTTT:[60]PCBM films (see Fig. 3b). The $20 \mathrm{kDa}$ fraction shows a very pronounced red-shifted vibronic progression, which is usually attributed to increased film crystallinity and $\pi$-stacking of polymer chains, ${ }^{72-74}$ while the $15 \mathrm{kDa}$ PBTTT absorption appears rather unstructured. The ENDOR spectra presented in Fig. 3a are measured on thick films, which dried from solution inside EPR quartz tubes under vacuum. This difference in preparation makes the direct comparison to spin coated thin films questionable as structure formation can critically depend on the drying time of the solvent. Therefore, we adapted a technique that is used e.g. for the sample preparation of conjugated polymer layers on transmission electron microscopy grids. PBTTT:[60]PCBM films were spin coated on PEDOT:PSS, floated in water, and wrapped around a thin $1 \mathrm{~mm}$ quartz tube which was inserted in the measurement tube and then evacuated and sealed under helium atmosphere as usual. In fact, the resulting ENDOR spectra measured on those thin films are identical to the ones measured on the thick films with considerable longer drying times (see ESI, $\dagger$ Fig. S3). This in turn means, that the electronic structure of the polaron is in our case independent of the preparation conditions. It also suggests, that the local chain morphology is retained and UV-vis measurements on thin films are applicable here in order to learn about the different degree of PBTTT backbone planarization due to $\pi$-stacking.

We can now conclude that the polaron delocalization in PBTTT $(15 \mathrm{kDa})$ is limited by structural disorder in the polymer layer, leading to an effective conjugation length close to the BTTT3 oligomer. On the other hand, the situation for PBTTT $(20 \mathrm{kDa})$ is not as clear since the spatial extent of the wave function, while more strongly delocalized, can still be limited by torsions along the backbone or alternatively by intrinsic decoherence mechanisms. Therefore, we now turn to a more detailed analysis of the oligomer ENDOR spectra in order to shed light on the different spectral contributions and the limiting mechanisms of charge delocalization. We also take advantage of the oligomer spectra to further test the adequacy of long-range corrected DFT functionals in assessing charge delocalization in $\pi$-conjugated systems.

\section{dBTTT1/BTTT1 ENDOR and influence of molecular conformation}

BTTT1 is the shortest and most simple molecule in this series, with six hydrogen nuclei directly bound to the aromatic ring system (two per each thiophene and two at the thienothiophene unit). Due to the symmetry of the molecule, it is sufficient to consider only the coupling of three inequivalent protons. The main spin density of the polaron is expected to be located along the $\pi$-orbital system of the conjugated backbone. However, the solubilizing side chains of BTTT1 also carry 13 protons per chain, which can give rise to the appearance of additional couplings in the ENDOR spectra. One usually expects only very weak charge density at the position of side chain protons because of the aliphatic nature of the side chains (also known as matrix contribution close to $a=0$ ). For this reason, side chains are usually omitted, truncated, or substituted by methyl groups when simulating ENDOR spectra with DFT. ${ }^{66}$ However, there are several examples of conjugated bio-molecules (such as flavines) where considerable polaron density is found at the side chain protons close to the aromatic units. ${ }^{75}$ The mechanism responsible for this effect is hyperconjugation; in the case of overlap/alignment of orbitals, charge is partially transferred from the $\mathrm{C}-\mathrm{H}$ side chain $\sigma$-bond to the $\mathrm{p}$-orbital of the $\mathrm{sp}^{2}$ hybridized carbon in the aromatic ring structure, which stabilizes the ground state due to increased spread of electron density. The transferred charge density $\rho$ at the position of the proton (and therefore the coupling strength $a_{\mathrm{H}}$ ) depends to a first approximation on dihedral angle $\varphi$ according to $a_{\mathrm{H}} \sim \cos ^{2}(\varphi),{ }^{76}$ which opens additional potential for structural investigations of organic radicals. This dependence, however, illustrates the potentially large influence of side chain proton couplings on ENDOR spectra for specific geometric arrangements (conformations). To exclude side chain couplings in our ENDOR spectra for a preliminary analysis and to quantify the contribution of those couplings depending on the conformation of the molecule, we synthesized the deuterated oligomer dBTTT1 where the side chain hydrogens atoms are replaced by deuterium (details on the synthesis can be found in the ESI $\dagger$ ). The difference in magnetic moment shifts the resonance position of the free deuteron by a factor of 6.5 to lower $\mathrm{RF}$ frequencies (at Q-band to approx. $8 \mathrm{MHz}$ ), which gives the possibility to investigate polaron couplings to the polaron to ${ }^{1} \mathrm{H}$ and ${ }^{2} \mathrm{H}$ nuclei separately in ENDOR spectra.

For a reliable simulation of ENDOR spectra, one has to consider specific suppression effects caused by the measurement technique itself. If the inversion pulse of the Davies sequence excites or inverts (due its bandwidth) both EPR transitions simultaneously (see Fig. 2), the ENDOR effect vanishes. Small couplings susceptible to this suppression effect arise most likely for distant $\mathrm{H}$ nuclei (non-conjugated side chains or molecular surrounding). While this suppression effect should result in vanishing ENDOR intensity at $\nu=0$, experimental ENDOR always show finite, but difficult to predict residual intensity. Consequently, we have chosen to assume complete suppression at $\nu=0$ in our simulations a Lorentzian line centered at $\nu=0$ with the width defined by to the excitation bandwidth (see above). Choosing increasingly longer microwave pulses would enhance the sensitivity for weakly coupled protons, but would also lead to an unwanted orientation selectivity rendering the spectra difficult to interpret. ${ }^{77}$ In the ESI $\dagger$ (Fig. S4) we show an example of the influence of the suppression effect on the expected simulated spectra for dBTTT1. 
a)

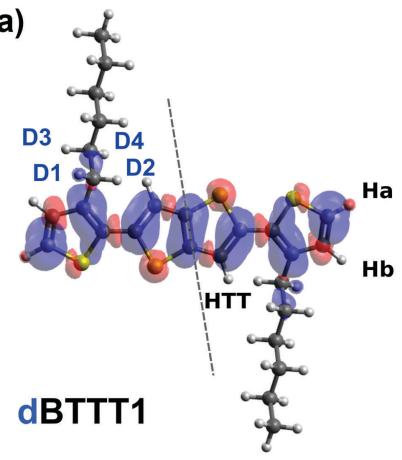

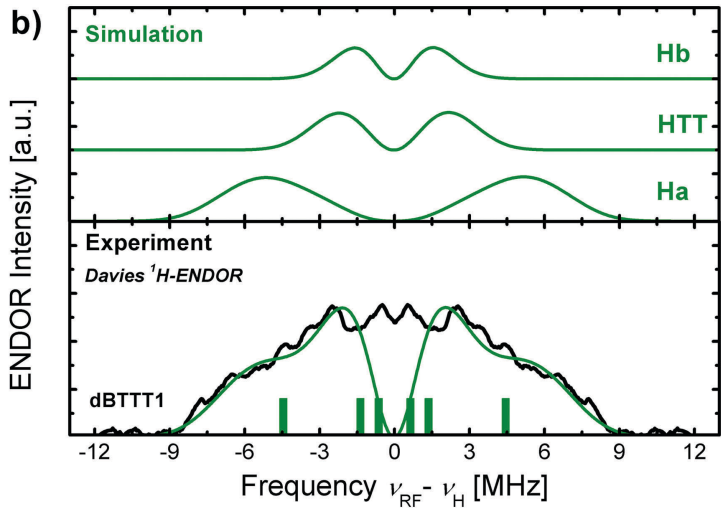

Fig. 4 (a) LC-BLYP DFT optimized chemical structure of the deuterated dBTTT1 cation in cis conformation with the side chain angle fixed to $105^{\circ}$ (taken from XRD measurements ${ }^{78}$ ). Also shown is the spin density of the positive polaron calculated at the LC-BLYP level of theory. (b) The graph shows a simulation of the dBTTT cation ${ }^{1} \mathrm{H}$ Davies ENDOR spectrum considering three aromatic protons $(\mathrm{Ha}, \mathrm{Hb}, \mathrm{HTT})$ visualizing contributions of the three different nuclei. The bottom graph shows the sum spectrum (green) in comparison to the experiment (black). Green bars represent the individual ${ }^{1} \mathrm{H}$ isotropic couplings used to simulate the spectrum. All simulated spectra include complete ENDOR effect suppression around $a=0$ (Davies inversion $\pi$-pulse $128 \mathrm{~ns})$.

Fig. 4a shows the chemical structure of the dBTTT1 oligomer with the aromatic hydrogens $\mathrm{Ha}, \mathrm{Hb}$ and HTT and the deuterium atoms D1, D2,.. etc. bound to the side chains. Recent X-ray diffraction investigations on the conformation of the nondeuterated oligomer have shown that the thiophene rings adapt a cis conformation with respect to the sulfur in the thienothiophene unit while the side chains arrange at an angle of $105^{\circ}$ with respect to the molecular plane. ${ }^{78}$ We use this structure as the basis for our simulations and optimize the geometry of the cation at the LC-BLYP/6-31G(d) level of theory while keeping the angle of the side chains fixed (even if this assumption were to be incorrect, we will later see that the arrangement of the side chains has only very little influence on the HFCs of $\mathrm{Ha}, \mathrm{Hb}$ and HTT). As we have recently shown, long-range corrected (LRC) functionals are essential in order to describe polaron delocalization in PBTTT. ${ }^{40}$ Here, we have chosen the LC-BLYP level of theory for all DFT calculations. Note that the differences between tuned LRC-functionals are in general small. However, in the present case, LC-BLYP seems to perform slightly better in comparison to the LC- $\omega \mathrm{PBE}$ and wB97X-D functionals, which were also used in the previous investigation (see $\mathrm{ESI} \dagger$ ).

Fig. 4a shows the LC-BLYP charge/spin density of the hole wave function, which is delocalized over the whole molecular backbone as well as the first $-\mathrm{CD}_{2}$ part of side chains. The highest coupling is to the outer hydrogen Ha while $\mathrm{Hb}$ and HTT show less spin density and therefore weaker coupling. We used the calculated isotropic and anisotropic HFC values to simulate the experimental ${ }^{1} \mathrm{H}$ ENDOR spectrum of the dBTTT cation (Fig. 4b) using the EPR software package easyspin. ${ }^{79}$ The simulated spectrum reproduces the shape of the experimental spectrum. Both weaker couplings to Hb and HTT are obviously affected by the suppression effect close to $a=0$ discussed above. Note that we do not observe a significant difference in the simulated HFC spectra between dBTTT in cis and trans configurations (see ESI, $\uparrow$ Fig. S5); thus, we cannot distinguish experimentally between the two forms.
The experimental data on dBTTT1 revealed the coupling of the polaron spin with the ${ }^{1} \mathrm{H}$ nuclei in the aromatic ring system. We now turn to the measurement and analysis of the coupling to the ${ }^{2} \mathrm{H}$ nuclei at the side chains of the oligomer. Together with appropriate simulations, we are able to check independently if the assumption on the side chain arrangement adapted from XRD on single crystals still holds for dBTTT1 mixed with PCBM. As pointed out earlier, the coupling to ${ }^{1} \mathrm{H}$ and ${ }^{2} \mathrm{H}$ can be investigated separately in ENDOR spectra because of the difference in the free Larmor frequency of the nuclei (factor of 6.5). Also, the ${ }^{2} \mathrm{H}$ ENDOR spectrum is narrowed by the same factor although the spin density at the position of the nucleus stays unchanged.

We calculated the HFC of the first four deuterium nuclei to the polaron spin in dBTTT as function of the side chain angle (see Fig. 5a) at the LC-BLYP level. The two ${ }^{2} \mathrm{H}$ close to the aromatic ring (D1, D2) exhibit the largest coupling, modulated by the expected $\cos ^{2}(\varphi)$ behavior. ${ }^{76}$ The maximum appears for parallel alignment of the $\mathrm{C}^{2} \mathrm{H}$ dihedral angle with the aromatic p-orbitals, and the minimum when the bond lies in the molecular plane. In coplanar arrangement of the side chains with the backbone, both $\mathrm{C}^{2} \mathrm{H}$ bonds point out of the plane to a higher degree than in the perpendicular case, which explains the different levels of the curve intersection points. For the more distant deuterium atoms 3 and 4, nearly no hyperconjugation (overlap of orbitals) takes place, which results in a very weak hole density and very little HFC at their positions.

The measurements of the ${ }^{2} \mathrm{H}$ ENDOR spectra (Fig. 5b) were performed with a slightly altered pulse sequence (MIMS ENDOR) that is especially sensitive to small hyperfine couplings. ${ }^{57,80}$ In contrast to Davies ENDOR, it does not produce a single central blind spot but several periodic blind spots depending on the specific pulse sequence time delay $\tau$. One can therefore reduce the width of the central suppression effect and resolve weaker couplings; on the other hand, this procedure induces additional blind spots at $1 /(2 \tau)$, which in our case are located at $\pm 1.66 \mathrm{MHz}$ 

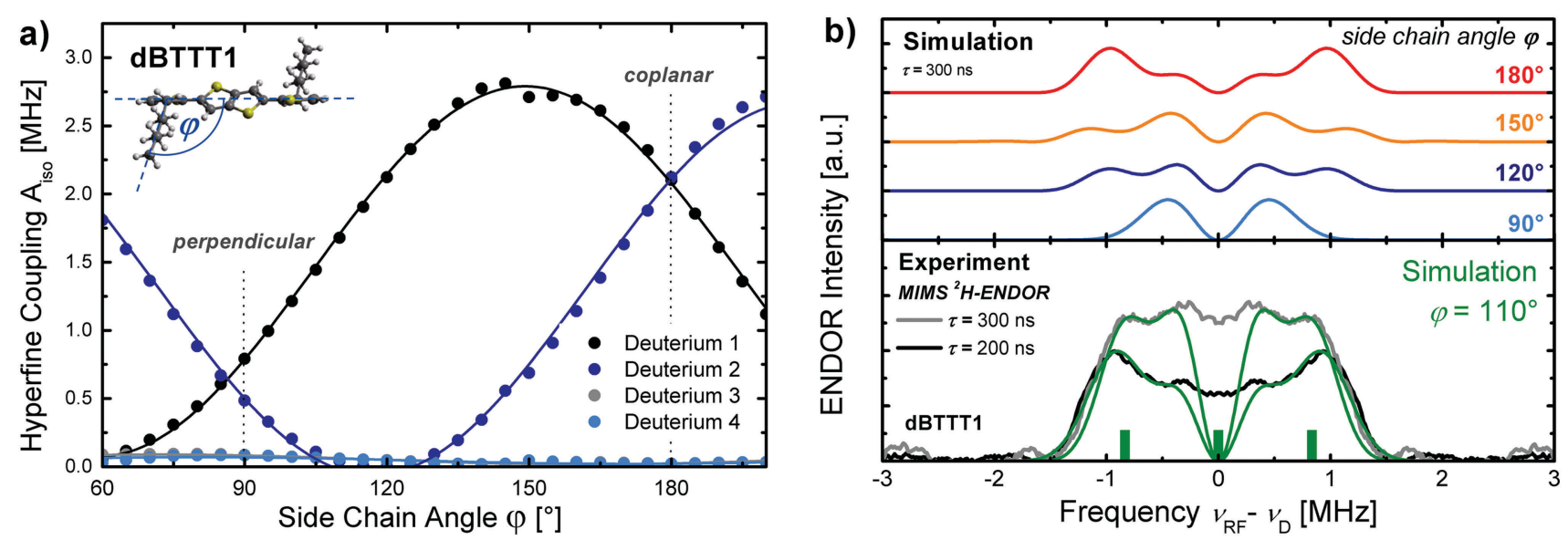

Fig. 5 (a) LC-BLYP-calculated isotropic hyperfine coupling strength for the first four deuterium nuclei in the dBTTT1 cation (cis conformation) as a function of the side chain angle. The lines show a guide to the eye considering a $\cos ^{2}(\varphi)$ dependence. (b) Top: Simulation of the ${ }^{2} H$ MIMS ENDOR spectrum with respect to the dihedral angle of the side chain (including ENDOR suppression for $\tau=300$ ns). Bottom: Experimental ${ }^{2} \mathrm{H}$ MIMS ENDOR spectra for $\tau=200 \mathrm{~ns}$ (black) and $300 \mathrm{~ns}$ (gray). Both are simulated (green) with the first four deuterium couplings and a constant side chain angle of $110^{\circ}$ (including the change in blind spot behavior depending on $\tau$ ). Green bars represent the individual ${ }^{2} \mathrm{H}$ isotropic couplings to simulate the spectrum.

( $\tau=300 \mathrm{~ns})$ and $\pm 2.5 \mathrm{MHz}(\tau=200 \mathrm{~ns})$, that is well separated from the experimental spectrum. The ${ }^{2} \mathrm{H}$ MIMS ENDOR spectrum with $\tau=300 \mathrm{~ns}$ (gray line) shows a distinct signature around $a / 2= \pm 0.85 \mathrm{MHz}$ which is slightly shifted to higher couplings for $\tau=200 \mathrm{~ns}$ due to the broadened blind spot at $a=0$. We used the ${ }^{2} \mathrm{H}$ HFC LC-BLYP calculations on the dBTTT cation presented in Fig. 5a to simulate several spectra as a function of the side chain angle with respect to the backbone (Fig. 5b). Taking the distinct visible $a / 2= \pm 0.85 \mathrm{MHz}$ transition as a basis for the description of the experimental results, the best fit is achieved by choosing a dihedral angle of $110^{\circ}$. The blind spot narrowing at $a=0$ vs. $\tau$ is well captured by our simulations and also reproduces the ostensible shift of the $a / 2= \pm 0.85 \mathrm{MHz}$ transition for $\tau=200 \mathrm{~ns}$. We note that due to the symmetry of the HFCs in Fig. 5 an angle of $70^{\circ}$ would simulate the experimental data equally well. However we exclude this possibility because the close proximity of the side chain to the TT unit appears to be energetically unfavorable. The peak at $a / 2= \pm 0.85 \mathrm{MHz}$ is caused by the $\mathrm{D} 1$ spin that carries considerable spin density in the $110^{\circ}$ side chain conformation (Fig. 4a). This value is in fact very close to recently published results on the XRD single crystal structure of BTTT1 $\left(105^{\circ}\right)$, which we used above for the simulation of the ${ }^{1} \mathrm{H}$ dBTTT ENDOR spectrum. We estimate the accuracy of this approach to be $\pm 5^{\circ}$ based on the onset of visible changes in the simulated spectral shape.

The dBTTT1 molecule enabled us to separately investigate and simulate the HFCs contributions of spin probes attached to the backbone (hydrogen) and distinguishable spin probes attached to the more flexible side chains (deuterium). Based on these results, we can now attempt to understand the complete ${ }^{1} \mathrm{H}$ BTTT ENDOR spectrum of the non-deuterated BTTT1 cation while this distinction could not be done without previous knowledge. Fig. 6 shows the corresponding ${ }^{1} \mathrm{H}$ ENDOR spectra of the dBTTT and BTTT1 cations in direct comparison. If the dihedral angle of the side chains based on previous

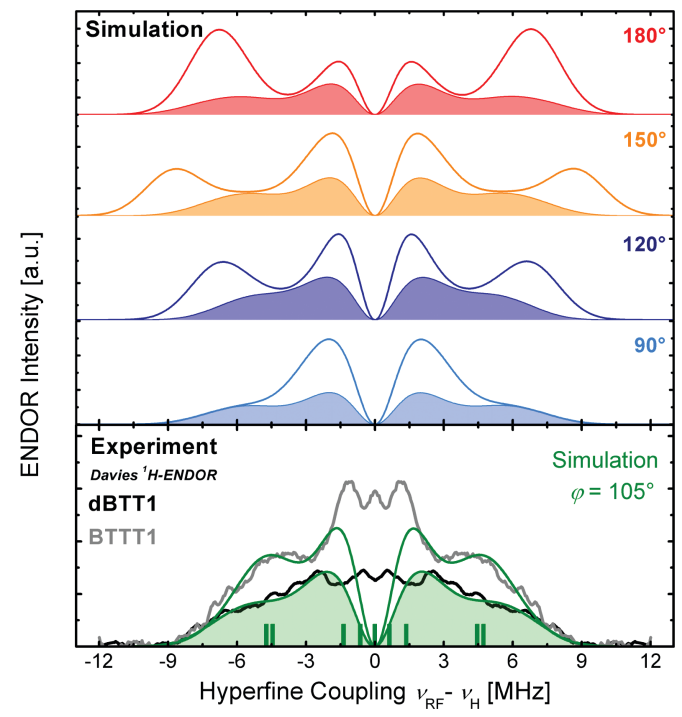

Fig. 6 The upper graph shows simulations of the BTTT1 cation ${ }^{1} \mathrm{H}$ Davies ENDOR spectra considering three aromatic protons $\mathrm{Ha}, \mathrm{Hb}, \mathrm{HTT}$ (shaded areas) and the complete spectrum (full lines) including side chain protons as function of the dihedral angle at the LC-BLYB level of theory. The lower graph shows a fit (green) of the experimental dBTTT1 spectrum (black) and non-deuterated BTTT1 spectrum (grey) with a constant side chain angle of $105^{\circ}$. Green bars represent the individual ${ }^{1} \mathrm{H}$ isotropic couplings to simulate the spectrum. All simulated spectra include complete ENDOR effect suppression around $a=0$ (Davies inversion $\pi$-pulse $128 \mathrm{~ns}$ ).

$\mathrm{X}$-ray diffraction measurements is preserved upon exchange of deuterium with hydrogen, one would expect additional ${ }^{1} \mathrm{H}$ couplings appearing at $a / 2= \pm 4.68 \mathrm{MHz}(6.5 \times 0.72 \mathrm{MHz}$ for $105^{\circ}$ ) and in the center of the spectrum. Without further simulation, this is clearly observed in the direct experimental comparison of both spectra. We performed again calculations of the HFCs with respect to the dihedral angle now consisting of ${ }^{1} \mathrm{H}$ couplings located in the aromatic backbone and in the side chains. Obviously the spin density at the position of the 
aromatic protons represented by the thin colored lines does not change significantly upon rotation of the side chains. The HFCs of the side chain protons $\mathrm{H} 1, \mathrm{H} 2, \mathrm{H} 3, \mathrm{H} 4$ appear with the same modulation as that presented in Fig. 5 and are added to the constant basis spectrum of $\mathrm{Ha}, \mathrm{Hb}$, and HTT. The best simulation of the experimental BTTT1 spectrum is reached by using the HFC values calculated on the molecular conformation of $105^{\circ}$. This value is not only the angle previously extracted from the single-crystal structure of BTTT1 but is also very close to the $110^{\circ}$ value we applied to simulate the ${ }^{2} \mathrm{H}$ MIMS ENDOR spectra of dBTTT1, which underlines the reliability and consistency of our approach.

Specific conformations of the BTTT1 molecule can in fact lead to a situation (e.g. side chain dihedral angle $150^{\circ}$ ) where the strongest coupling visible in the ENDOR spectrum is not given by a proton in the conjugated backbone but in the aliphatic side chain. It is now clear that ${ }^{1} \mathrm{H}$ side chain couplings can significantly contribute to ENDOR spectra of organic conjugated molecules or polymers particularly for selected molecular arrangements. As a main drawback, comparisons of experimental results with simulated ENDOR spectra based on DFT calculations are questionable when the morphologic arrangement is unknown or the side chains are not treated adequately (e.g., they are just truncated to methyl groups). Since the availability of deuterated molecules that help to separate those influences experimentally is limited, reliable simulation of ENDOR spectra needs to be combined with independent structural investigations on the organic compounds (e.g., XRD, NEXAFS, solid-state NMR, and/or IR spectroscopy). After this detailed analysis of the BTTT1 monomer, we now apply what we have learned to the BTTT2 and BTTT3 oligomers.

\section{BTTT2 ENDOR}

An adequate simulation of the BTTT2 cation ${ }^{1} \mathrm{H}$ ENDOR spectrum therefore has to rely on previous investigations of the molecular structure in thin films. Recent investigations by XRD on single crystals showed that the BTTT2 side chains are coplanar with the conjugated backbone, thereby leaving enough space to host PCBM. In the blend with PCBM, these channels are maintained and enable the co-crystallization and intercalation of PCBM. ${ }^{41}$ Note that for the co-crystalization of PBTTT and PCBM a different arrangement of side chains has been deduced (vide infra BTTT3). ${ }^{81}$ On that basis the HFCs were calculated on optimized BTTT2 cation geometries (constraining the side chains to coplanar arrangement) to simulate the ${ }^{1}$ H BTTT2 ENDOR spectrum (Fig. 7b). The hyperfine coupling to aromatic protons (thin lines), which is insensitive to the side chain arrangement, gives first evidence on the match between experiment and simulation. Adding the HFC of the side chain protons instantly visualizes that the major part of the BTTT2 ENDOR spectrum consists of contributions that strongly depend on the geometric arrangement of the side chains, which again stresses the importance of the side chain couplings. The match between simulation and experiment is however not perfect. Due to the increasing complexity of the larger oligomers, a full simulation of the dependence on the dihedral angle, comparable to what we have reported for ABTTT1, would be, however, computationally challenging. We note that a larger deviation (e.g. perpendicular arrangement) would shift a significant part of the spectrum to the region between $0-1 \mathrm{MHz}$, which is not visible in the experimental spectrum (see Fig. 5a). We therefore suggest that minor deviations from the coplanar arrangement can exist in films of BTTT2 mixed with [60]PCBM.

Structural information on the BTTT3 oligomer is even more limited as it is not possible to deduce a complete crystallographic model from previous XRD measurements. Grazing incidence $\mathrm{X}$-ray diffraction on thin oligomer films, however, shows that typical stacking distances along the different crystallographic directions already match those for PBTTT films, with the main changes in structure formation occurring between BTTT1 and BTTT3. Consequently, it is reasonable to rely on a number of investigations characterizing the intercalation of PBTTT with PCBM. Studies based on IR, NEXAFS, and XRD suggest for pure PBTTT layers a tilt angle of the stretched side chains by $24-40^{\circ}$ in view along the backbone axis. ${ }^{82,83} \mathrm{~A}$ more recent investigation additionally incorporating solid state NMR and molecular a)

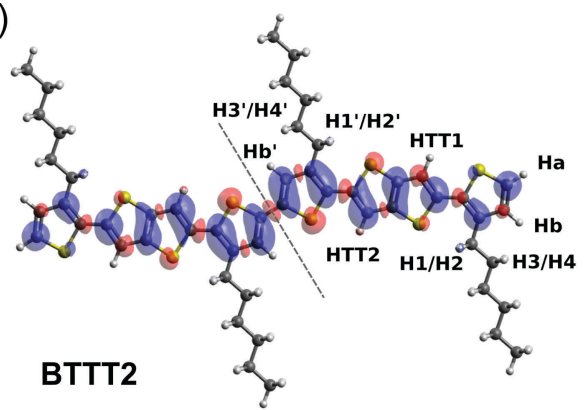

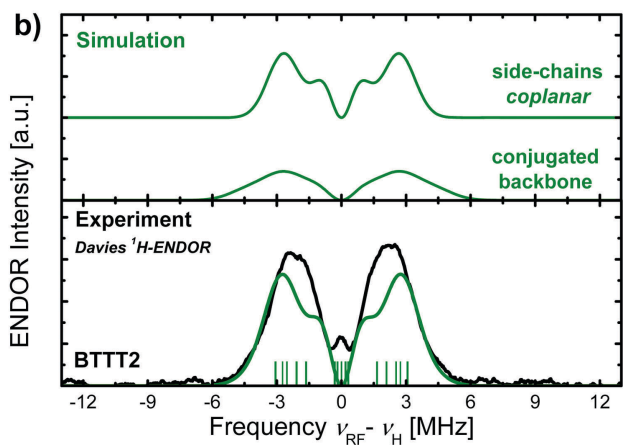

Fig. 7 (a) LC-BLYP optimized chemical structure of the BTTT2 cation with side chains constrained to a coplanar arrangement (in reference to XRD measurements ${ }^{41}$ ). Also shown is the charge/spin density of the positive polaron. (b) The upper graph shows separate simulations of the BTT2 cation ${ }^{1} \mathrm{H}$ Davies ENDOR spectra considering five aromatic protons $\mathrm{Ha}, \mathrm{Hb}, \mathrm{HTT} 1, \mathrm{HTT2}, \mathrm{Hb}^{\prime}$ and the HFC caused by side chain protons in coplanar arrangement. The lower graph shows the complete simulation (green) of the experimental BTTT2 spectrum (black) with LC-BLYP. Green bars represent the individual ${ }^{1} \mathrm{H}$ isotropic couplings to simulate the spectrum. All simulated spectra include complete ENDOR effect suppression around $a=0$ (Davies inversion $\pi$-pulse $128 \mathrm{~ns}$ ). 
a)

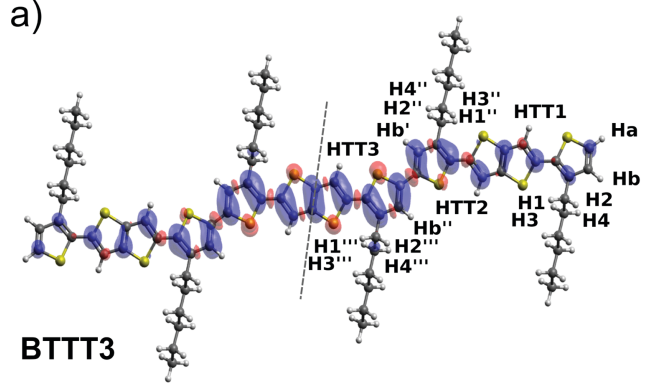

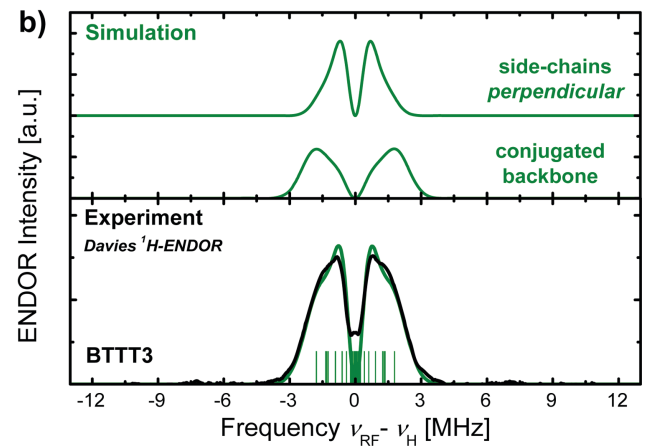

Fig. 8 (a) LC-BLYP optimized chemical structure of the BTTT3 cation with side chains constrained to a nearly perpendicular arrangement (analog to PBTTT as described in the main text ${ }^{81}$ ). The spin density of the positive polaron is visualized. (b) The upper graph shows separate simulations of BTTT3 cation ${ }^{1} \mathrm{H}$ Davies ENDOR spectra considering seven aromatic protons $\mathrm{Ha}, \mathrm{Hb}, \mathrm{Hb}$, $\mathrm{Hb}$ ", HTT1, HTT2, HTT3 and the HFC caused by side chain protons in nearly perpendicular arrangement. The lower graph shows the complete simulation (green) of the experimental BTTT3 spectrum (black) with LC-BLYP. Green bars represent the individual ${ }^{1} \mathrm{H}$ isotropic couplings to simulate the spectrum. All simulated spectra include complete ENDOR effect suppression around $a=0$ (Davies inversion $\pi$-pulse 128 ns).

dynamics data implies that this tilt angle is retained in a mixture with PCBM. ${ }^{81}$ Based on the nomenclature introduced in Fig. 5a, PBTTT side chains arrange in a nearly perpendicular fashion $\left(90^{\circ}\right)$ as shown in Fig. 8 a in the case of BTTT3. We performed a DFT optimization for the geometry of the BTTT3 cation with this constraint and again evaluated the HFC of all hydrogen spins with the polaron. The results of the ${ }^{1} \mathrm{H}$ Davies ENDOR spectra simulation are shown in Fig. 8b. A first comparison, focusing on the conformationally more insensitive ${ }^{1} \mathrm{H}$ spins bound to the conjugated backbone (see Fig. 6), reveals that those mainly contribute larger couplings to the BTTT3 spectrum. The inclusion of the HFC of the side chain protons in the simulation leads to a robust description of the complete BTTT3 ENDOR spectrum by adding weaker couplings due to the perpendicular arrangement. It is obvious that any other molecular conformation of the side chains than that depicted in Fig. 8a would lead to the appearance of larger couplings in the spectra, according to the $\cos ^{2}(\varphi)$ behavior. Assuming that the BTTT3 conformation is very similar to PBTTT therefore seems very reasonable.

\section{Conclusion}

We have shown that considerable care has to be taken for deducing polaron localization lengths from hyperfine coupling spectra of conjugated polymers. This is especially true when investigating ${ }^{1} \mathrm{H}$ couplings, which may consist to a significant degree of couplings to protons in the non-conjugated side chains. Those couplings are, however, very sensitive to the structural arrangement with respect to the conjugated backbone. Therefore, reliable simulations of conjugated polymer ENDOR spectra need to be combined with independent structural investigations on the organic compounds. In our case, we have been able to take advantage of the PBTTT oligomer series and the deuterated molecule dBTTT1 to disentangle these different contributions to the ENDOR spectra. On that basis, we can directly draw conclusions on the extent of the polaron wavefunction in PBTTT as function of the film morphology.

\section{PBTTT polaron delocalization}
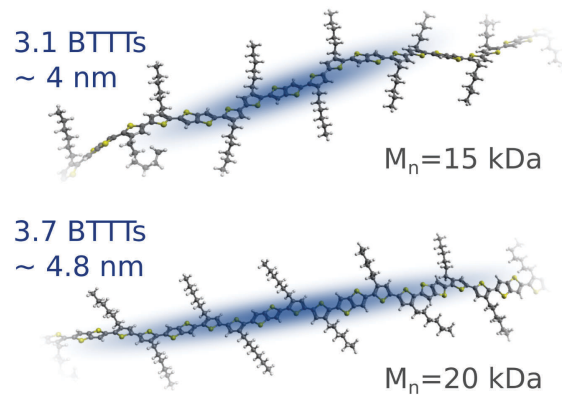

Fig. 9 Schematic representation of the polaron delocalization length in the two PBTTT samples with different molecular weights.

The consistent simulation of the individual oligomer spectra confirms that long-range corrected DFT functionals can properly describe the change in coupling strength with increasing oligomer size. For shorter oligomers as BTTT1/BTTT2, B3LYP seems to work comparably well as both B3LYP and LC-BLYP reproduce the $1 / N$ behavior of the calculated maximum HFC in this oligomer length regime (see ESI, $\dagger$ Fig. S9). However, since B3LYP tends to overdelocalize wave functions, it start failing on longer oligomers where LC-BLYP predicts a limited polaron size and saturation of the HFC. We can therefore compare the experimental results on two PBTTT samples of different molecular weight, to the polaron polymer limit deduced from long-range corrected DFT calculations. In the lower molecular weight sample, the delocalization of the polaron extends over 3.1 BTTT units, that is about over 12 thiophene rings; it is clearly limited by structural disorder, leading to twists and kinks along the polymer backbone that break the conjugation (Fig. 9). Since such defects are difficult to model a priori with DFT, calculations on rather disordered or amorphous conjugated polymers might overestimate the actual polaron localization length.

The main reason for the farther delocalization of the hole in higher molecular weight PBTTT films likely is the increased degree of order, which leads in this particular case to a polaron 
size of 3.7 BTTT units, that is, over about 15 thiophene rings. Previous DFT calculations on BTTT cations have shown that saturation of polaron delocalization starts at a length of BTTT4, ${ }^{40}$ which is very close to the maximum experimental value measured here for the $20 \mathrm{kD} M_{\mathrm{n}}$ PBTTT. This implies that in highly ordered PBTTT films positive polarons can reach their intrinsic delocalization limit, which matches that predicted by DFT calculations. Our results underline the importance of achieving planarization of the polymer backbone in order to reach a high degree of polaron delocalization and to maximize charge transport.

To summarize, we exploited ENDOR techniques to measure reliably polaron delocalization in PBTTT samples and to correlate the extent of delocalization with the polymer film morphology. Our study forms a useful basis for further studies on the hyperfine coupling spectra of conjugated polymers. Polaron delocalization is expected to have a strong influence on phenomena such as charge transport and charge generation in functional organic devices. However, at this time, a convincing experimental correlation between these processes and charge delocalization remains missing.

\section{Acknowledgements}

The authors like to thank for financial support from the DFG (SPP 1601) and the Helmholtz Association (Energie-Allianz Hybrid-Photovoltaik). This work has been partly supported by King Abdullah University of Science and Technology (KAUST). We acknowledge the KAUST IT Research Computing Team for providing computational and storage resources and thank Dr Cheng Zhong, Dr Haitao Sun, and Dr Bradley D. Rose for stimulating discussions. A. L. B. acknowledges the National Science Foundation (DMR-1508627).

\section{References}

1 J. L. Bredas and G. B. Street, Polarons, bipolarons, and solitons in conducting polymers, Acc. Chem. Res., 1985, 18(10), 309-315.

2 H. Bässler, Charge Transport in Disordered Organic Photoconductors a Monte Carlo Simulation Study, Phys. Status Solidi B, 1993, 175(1), 15-56.

3 N. Tessler, Y. Preezant, N. Rappaport and Y. Roichman, Charge Transport in Disordered Organic Materials and Its Relevance to Thin-Film Devices: A Tutorial Review, Adv. Mater., 2009, 21(27), 2741-2761.

4 S. D. Baranovskii, Theoretical description of charge transport in disordered organic semiconductors, Phys. Status Solidi B, 2014, 251(3), 487-525.

5 J.-L. Brédas, D. Beljonne, V. Coropceanu and J. Cornil, Charge-Transfer and Energy-Transfer Processes in $\pi$-Conjugated Oligomers and Polymers: A Molecular Picture, Chem. Rev., 2004, 104(11), 4971-5004.

6 S. T. Hoffmann, F. Jaiser, A. Hayer, H. Bässler, T. Unger, S. Athanasopoulos, D. Neher and A. Köhler, How Do Disorder, Reorganization, and Localization Influence the
Hole Mobility in Conjugated Copolymers?, J. Am. Chem. Soc., 2013, 135(5), 1772-1782.

7 R. Steyrleuthner, R. Di Pietro, B. A. Collins, F. Polzer, S. Himmelberger, M. Schubert, Z. Chen, S. Zhang, A. Salleo, H. Ade, A. Facchetti and D. Neher, The Role of Regioregularity, Crystallinity, and Chain Orientation on Electron Transport in a High-Mobility n-Type Copolymer, J. Am. Chem. Soc., 2014, 136(11), 4245-4256.

8 D. Venkateshvaran, M. Nikolka, A. Sadhanala, V. Lemaur, M. Zelazny, M. Kepa, M. Hurhangee, A. J. Kronemeijer, V. Pecunia, I. Nasrallah, I. Romanov, K. Broch, I. McCulloch, D. Emin, Y. Olivier, J. Cornil, D. Beljonne and H. Sirringhaus, Approaching disorder-free transport in high-mobility conjugated polymers, Nature, 2014, 515(7527), 384-388.

9 G. Kim, S.-J. Kang, G. K. Dutta, Y.-K. Han, T. J. Shin, Y.-Y. Noh and C. Yang, A Thienoisoindigo-Naphthalene Polymer with Ultrahigh Mobility of $14.4 \mathrm{~cm}^{2} \mathrm{~V}^{-1} \mathrm{~s}^{-1}$ That Substantially Exceeds Benchmark Values for Amorphous Silicon Semiconductors, J. Am. Chem. Soc., 2014, 136(26), 9477-9483.

10 I. Kang, H.-J. Yun, D. S. Chung, S.-K. Kwon and Y.-H. Kim, Record High Hole Mobility in Polymer Semiconductors via Side-Chain Engineering, J. Am. Chem. Soc., 2013, 135(40), 14896-14899.

11 X. Zhang, H. Bronstein, A. J. Kronemeijer, J. Smith, Y. Kim, R. J. Kline, L. J. Richter, T. D. Anthopoulos, H. Sirringhaus, K. Song, M. Heeney, W. Zhang, I. McCulloch and D. M. DeLongchamp, Molecular origin of high field-effect mobility in an indacenodithiophene-benzothiadiazole copolymer, Nat. Commun., 2013, 4.

12 X. Zhang, L. J. Richter, D. M. DeLongchamp, R. J. Kline, M. R. Hammond, I. McCulloch, M. Heeney, R. S. Ashraf, J. N. Smith, T. D. Anthopoulos, B. Schroeder, Y. H. Geerts, D. A. Fischer and M. F. Toney, Molecular Packing of HighMobility Diketo Pyrrolo-Pyrrole Polymer Semiconductors with Branched Alkyl Side Chains, J. Am. Chem. Soc., 2011, 133(38), 15073-15084.

13 W. Zhang, J. Smith, S. E. Watkins, R. Gysel, M. McGehee, A. Salleo, J. Kirkpatrick, S. Ashraf, T. Anthopoulos, M. Heeney and I. McCulloch, Indacenodithiophene Semiconducting Polymers for High-Performance, Air-Stable Transistors, J. Am. Chem. Soc., 2010, 132(33), 11437-11439.

14 J. S. Ha, K. H. Kim and D. H. Choi, 2,5-Bis(2-octyldodecyl)pyrrolo[3,4-c]pyrrole-1,4-(2H,5H)-dione-Based Donor-Acceptor Alternating Copolymer Bearing 5,5'-Di(thiophen-2-yl)-2,2'biselenophene Exhibiting $1.5 \mathrm{~cm}^{2} \mathrm{~V}^{-1} \mathrm{~s}^{-1}$ Hole Mobility in Thin-Film Transistors, J. Am. Chem. Soc., 2011, 133(27), 10364-10367.

15 H. Chen, Y. Guo, G. Yu, Y. Zhao, J. Zhang, D. Gao, H. Liu and Y. Liu, Highly $\pi$-Extended Copolymers with Diketopyrrolopyrrole Moieties for High-Performance Field-Effect Transistors, Adv. Mater., 2012, 24(34), 4618-4622.

16 Z. Chen, P. Cai, J. Chen, X. Liu, L. Zhang, L. Lan, J. Peng, Y. Ma and Y. Cao, Low Band-Gap Conjugated Polymers with Strong Interchain Aggregation and Very High Hole Mobility Towards Highly Efficient Thick-Film Polymer Solar Cells, Adv. Mater., 2014, 26(16), 2586-2591. 
17 P. Sonar, S. P. Singh, Y. Li, M. S. Soh, A. Dodabalapur and A. Low-Bandgap Diketopyrrolopyrrole-BenzothiadiazoleBased, Copolymer for High-Mobility Ambipolar Organic Thin-Film Transistors, Adv. Mater., 2010, 22(47), 5409-5413.

18 S. Schott, E. Gann, L. Thomsen, S.-H. Jung, J.-K. Lee, C. R. McNeill and H. Sirringhaus, Charge-Transport Anisotropy in a Uniaxially Aligned Diketopyrrolopyrrole-Based Copolymer, Adv. Mater., 2015, 27(45), 7356-7364.

19 H.-R. Tseng, H. Phan, C. Luo, M. Wang, L. A. Perez, S. N. Patel, L. Ying, E. J. Kramer, T.-Q. Nguyen, G. C. Bazan and A. J. Heeger, High-Mobility Field-Effect Transistors Fabricated with Macroscopic Aligned Semiconducting Polymers, Adv. Mater., 2014, 26(19), 2993-2998.

20 B. B.-Y. Hsu, C.-M. Cheng, C. Luo, S. N. Patel, C. Zhong, H. Sun, J. Sherman, B. H. Lee, L. Ying, M. Wang, G. Bazan, M. Chabinyc, J.-L. Brédas and A. Heeger, The Density of States and the Transport Effective Mass in a Highly Oriented Semiconducting Polymer: Electronic Delocalization in 1D, Adv. Mater., 2015, 27(47), 7759-7765.

21 A. A. Bakulin, A. Rao, V. G. Pavelyev, P. H. M. van Loosdrecht, M. S. Pshenichnikov, D. Niedzialek, J. Cornil, D. Beljonne and R. H. Friend, The Role of Driving Energy and Delocalized States for Charge Separation in Organic Semiconductors, Science, 2012, 335(6074), 1340-1344.

22 G. Grancini, M. Maiuri, D. Fazzi, A. Petrozza, H. J. Egelhaaf, D. Brida, G. Cerullo and G. Lanzani, Hot exciton dissociation in polymer solar cells, Nat. Mater., 2013, 12(1), 29-33.

23 D. Caruso and A. Troisi, Long-range exciton dissociation in organic solar cells, Proc. Natl. Acad. Sci. U. S. A., 2012, 109(34), 13498-13502.

24 B. M. Savoie, A. Rao, A. A. Bakulin, S. Gelinas, B. Movaghar, R. H. Friend, T. J. Marks and M. A. Ratner, Unequal Partnership: Asymmetric Roles of Polymeric Donor and Fullerene Acceptor in Generating Free Charge, J. Am. Chem. Soc., 2014, 136(7), 2876-2884.

25 S. Gélinas, A. Rao, A. Kumar, S. L. Smith, A. W. Chin, J. Clark, T. S. van der Poll, G. C. Bazan and R. H. Friend, Ultrafast Long-Range Charge Separation in Organic Semiconductor Photovoltaic Diodes, Science, 2014, 343(6170), 512-516.

26 S. Few, J. M. Frost and J. Nelson, Models of charge pair generation in organic solar cells, Phys. Chem. Chem. Phys., 2015, 17(4), 2311-2325.

27 B. H. Hamadani, D. J. Gundlach, I. McCulloch and M. Heeney, Undoped polythiophene field-effect transistors with mobility of $1 \mathrm{~cm}^{2} \mathrm{~V}^{-1} \mathrm{~s}^{-1}$, Appl. Phys. Lett., 2007, 91(24), 243512.

28 R. J. Kline, D. M. DeLongchamp, D. A. Fischer, E. K. Lin, M. Heeney, I. McCulloch and M. F. Toney, Significant dependence of morphology and charge carrier mobility on substrate surface chemistry in high performance polythiophene semiconductor films, Appl. Phys. Lett., 2007, 90(6), 062117.

29 J. Rivnay, R. Noriega, J. E. Northrup, R. J. Kline, M. F. Toney and A. Salleo, Structural origin of gap states in semicrystalline polymers and the implications for charge transport, Phys. Rev. B: Condens. Matter Mater. Phys., 2011, 83(12), 121306.
30 M. J. Lee, D. Gupta, N. Zhao, M. Heeney, I. McCulloch and H. Sirringhaus, Anisotropy of Charge Transport in a Uniaxially Aligned and Chain-Extended, High-Mobility, Conjugated Polymer Semiconductor, Adv. Funct. Mater., 2011, 21(5), 932-940.

31 C. Wang, L. H. Jimison, L. Goris, I. McCulloch, M. Heeney, A. Ziegler and A. Salleo, Microstructural Origin of High Mobility in High-Performance Poly(thieno-thiophene) Thin-Film Transistors, Adv. Mater., 2010, 22(6), 697-701.

32 X. Zhang, S. D. Hudson, D. M. DeLongchamp, D. J. Gundlach, M. Heeney and I. McCulloch, In-Plane Liquid Crystalline Texture of High-Performance Thienothiophene Copolymer Thin Films, Adv. Funct. Mater., 2010, 20(23), 4098-4106.

33 T. Schuettfort, B. Watts, L. Thomsen, M. Lee, H. Sirringhaus and C. R. McNeill, Microstructure of Polycrystalline PBTTT Films: Domain Mapping and Structure Formation, ACS Nano, 2012, 6(2), 1849-1864.

34 S. Himmelberger, J. Dacuña, J. Rivnay, L. H. Jimison, T. McCarthy-Ward, M. Heeney, I. McCulloch, M. F. Toney and A. Salleo, Effects of Confinement on Microstructure and Charge Transport in High Performance Semicrystalline Polymer Semiconductors, Adv. Funct. Mater., 2013, 23(16), 2091-2098.

35 L. Biniek, N. Leclerc, T. Heiser, R. Bechara and M. Brinkmann, Large Scale Alignment and Charge Transport Anisotropy of pBTTT Films Oriented by High Temperature Rubbing, Macromolecules, 2013, 46(10), 4014-4023.

36 G. Giri, D. M. DeLongchamp, J. Reinspach, D. A. Fischer, L. J. Richter, J. Xu, S. Benight, A. Ayzner, M. He, L. Fang, G. Xue, M. F. Toney and Z. Bao, Effect of Solution Shearing Method on Packing and Disorder of Organic Semiconductor Polymers, Chem. Mater., 2015, 27(7), 2350-2359.

37 B. A. Collins, J. E. Cochran, H. Yan, E. Gann, C. Hub, R. Fink, C. Wang, T. Schuettfort, C. R. McNeill, M. L. Chabinyc and H. Ade, Polarized X-ray scattering reveals non-crystalline orientational ordering in organic films, Nat. Mater., 2012, 11(6), 536-543.

38 M. L. Chabinyc, M. F. Toney, R. J. Kline, I. McCulloch and M. Heeney, X-ray Scattering Study of Thin Films of Poly(2,5-bis(3-alkylthiophen-2-yl)thieno[3,2-b]thiophene), J. Am. Chem. Soc., 2007, 129(11), 3226-3237.

39 T. Liu and A. Troisi, Understanding the Microscopic Origin of the Very High Charge Mobility in PBTTT: Tolerance of Thermal Disorder, Adv. Funct. Mater., 2014, 24(7), 925-933.

40 Y. Zhang, R. Steyrleuthner and J.-L. Bredas, Charge Delocalization in Oligomers of Poly(2,5-bis(3-alkylthiophene-2yl)thieno[3,2-b]thiophene) (РВTTT), J. Phys. Chem. C, 2016, 120(18), 9671-9677.

41 L. Zhang, F. Liu, Y. Diao, H. S. Marsh, N. S. Colella, A. Jayaraman, T. P. Russell, S. C. B. Mannsfeld and A. L. Briseno, The Good Host: Formation of Discrete OneDimensional Fullerene "Channels" in Well-Ordered Poly(2,5bis(3-alkylthiophen-2-yl)thieno[3,2-b]thiophene) Oligomers, J. Am. Chem. Soc., 2014, 136(52), 18120-18130.

42 R. Noriega, J. Rivnay, K. Vandewal, F. P. V. Koch, N. Stingelin, P. Smith, M. F. Toney and A. Salleo, A general 
relationship between disorder, aggregation and charge transport in conjugated polymers, Nat. Mater., 2013, 12(11), 1038-1044.

43 D. Beljonne, J. Cornil, H. Sirringhaus, P. J. Brown, M. Shkunov, R. H. Friend and J. L. Brédas, Optical Signature of Delocalized Polarons in Conjugated Polymers, Adv. Funct. Mater., 2001, 11(3), 229-234.

44 G. Heimel, The Optical Signature of Charges in Conjugated Polymers, ACS Cent. Sci., 2016, 2(5), 309-315.

45 R. Österbacka, C. P. An, X. M. Jiang and Z. V. Vardeny, Two-Dimensional Electronic Excitations in Self-Assembled Conjugated Polymer Nanocrystals, Science, 2000, 287(5454), 839-842.

46 R. Ghosh, C. M. Pochas and F. C. Spano, Polaron Delocalization in Conjugated Polymer Films, J. Phys. Chem. C, 2016, 120(21), 11394-11406.

47 M. Wohlgenannt, X. M. Jiang and Z. V. Vardeny, Confined and delocalized polarons in $\pi$-conjugated oligomers and polymers: A study of the effective conjugation length, Phys. Rev. B: Condens. Matter Mater. Phys., 2004, 69(24), 241204.

48 L. Zaikowski, P. Kaur, C. Gelfond, E. Selvaggio, S. Asaoka, Q. Wu, H.-C. Chen, N. Takeda, A. R. Cook, A. Yang, J. Rosanelli and J. R. Miller, Polarons, Bipolarons, and Side-By-Side Polarons in Reduction of Oligofluorenes, J. Am. Chem. Soc., 2012, 134(26), 10852-10863.

49 C. Wiebeler, R. Tautz, J. Feldmann, E. von Hauff, E. Da Como and S. Schumacher, Spectral Signatures of Polarons in Conjugated Co-polymers, J. Phys. Chem. B, 2013, 117(16), 4454-4460.

50 N. Takeda and J. R. Miller, Poly(3-decylthiophene) Radical Anions and Cations in Solution: Single and Multiple Polarons and Their Delocalization Lengths in Conjugated Polymers, J. Phys. Chem. B, 2012, 116(50), 14715-14723.

51 S. Kahmann, D. Fazzi, G. J. Matt, W. Thiel, M. A. Loi and C. J. Brabec, Polarons in Narrow Band Gap Polymers Probed over the Entire Infrared Range: A Joint Experimental and Theoretical Investigation, J. Phys. Lett., 2016, 7(22), 4438-4444.

52 J. R. Norris, R. A. Uphaus, H. L. Crespi and J. J. Katz, Electron Spin Resonance of Chlorophyll and the Origin of Signal I in Photosynthesis, Proc. Natl. Acad. Sci. U. S. A., 1971, 68(3), 625-628.

53 G. Feher, A. J. Hoff, R. A. Isaacson and L. C. Ackerson, Endor Experiments On Chlorophyll And Bacteriochlorophyll In Vitro And In The Photosynthetic Unit, Ann. N. Y. Acad. Sci., 1975, 244(1), 239-259.

54 F. Lendzian, M. Huber, R. A. Isaacson, B. Endeward, M. Plato, B. Bönigk, K. Möbius, W. Lubitz and G. Feher, The electronic structure of the primary donor cation radical in Rhodobacter sphaeroides R-26: ENDOR and TRIPLE resonance studies in single crystals of reaction centers, Biochim. Biophys. Acta, Bioenerg., 1993, 1183(1), 139-160.

55 N. S. Sariciftci, L. Smilowitz, A. J. Heeger and F. Wudl, Photoinduced Electron Transfer from a Conducting Polymer to Buckminsterfullerene, Science, 1992, 258(5087), 1474-1476.
56 F. Kraffert, R. Steyrleuthner, S. Albrecht, D. Neher, M. C. Scharber, R. Bittl and J. Behrends, Charge Separation in PCPDTBT:PCBM Blends from an EPR Perspective, J. Phys. Chem. C, 2014, 118(49), 28482-28493.

57 C. Gemperle and A. Schweiger, Pulsed electron-nuclear double resonance methodology, Chem. Rev., 1991, 91(7), 1481-1505.

58 Y. Shimoi, S. Abe, S.-i. Kuroda and K. Murata, Polarons and their ENDOR spectra in poly( $p$-phenylene vinylene), Solid State Commun., 1995, 95(3), 137-141.

59 P. Brendel, A. Grupp, M. Mehring, R. Schenk, K. Müllen and W. Huber, Chain length-dependent polaron $\left(\mathrm{P}^{-}\right)$width in phenylenevinylene oligomers: a solid state ENDOR investigation, Synth. Met., 1991, 45(1), 49-57.

60 S. Kuroda, K. Marumoto, H. Ito, N. C. Greenham, R. H. Friend, Y. Shimoi and S. Abe, Spin distributions and excitation spectra of optically generated polarons in poly( $p$-phenylenevinylene) derivatives, Chem. Phys. Lett., 2000, 325(1-3), 183-188.

61 K. Marumoto, N. Takeuchi and S. Kuroda, Nanoscale spatial extent of photogenerated polarons in regioregular poly(3octylthiophene), Chem. Phys. Lett., 2003, 382(5-6), 541-546.

62 S.-i. Kuroda, K. Marumoto, T. Sakanaka, N. Takeuchi, Y. Shimoi, S. Abe, H. Kokubo and T. Yamamoto, Electronnuclear double-resonance observation of spatial extent of polarons in polythiophene and poly(3-alkylthiophene), Chem. Phys. Lett., 2007, 435(4-6), 273-277.

63 A. Aguirre, P. Gast, S. Orlinskii, I. Akimoto, E. J. J. Groenen, H. El Mkami, E. Goovaerts and S. Van Doorslaer, Multifrequency EPR analysis of the positive polaron in I2-doped poly(3-hexylthiophene) and in poly[2-methoxy-5-(3,7dimethyloctyloxy)]-1,4-phenylenevinylene, Phys. Chem. Chem. Phys., 2008, 10(47), 7129-7138.

64 K. Marumoto, M. Kato, H. Kondo, S. Kuroda, N. C. Greenham, R. H. Friend, Y. Shimoi and S. Abe, Electron spin resonance and electron nuclear double resonance of photogenerated polarons in polyfluorene and its fullerene composite, Phys. Rev. B: Condens. Matter Mater. Phys., 2009, 79(24), 245204.

65 Y. Ling, S. Van Mierloo, A. Schnegg, M. Fehr, P. Adriaensens, L. Lutsen, D. Vanderzande, W. Maes, E. Goovaerts and S. Van Doorslaer, Electronic structure of positive and negative polarons in functionalized dithienylthiazolo[5,4- $d]$ thiazoles: a combined EPR and DFT study, Phys. Chem. Chem. Phys., 2014, 16(21), 10032-10040.

66 J. Niklas, K. L. Mardis, B. P. Banks, G. M. Grooms, A. Sperlich, V. Dyakonov, S. Beaupre, M. Leclerc, T. Xu, L. Yu and O. G. Poluektov, Highly-efficient charge separation and polaron delocalization in polymer-fullerene bulkheterojunctions: a comparative multi-frequency EPR and DFT study, Phys. Chem. Chem. Phys., 2013, 15(24), 9562-9574.

67 T. Körzdörfer, J. S. Sears, C. Sutton and J.-L. Brédas, Long-range corrected hybrid functionals for $\pi$-conjugated systems: dependence of the range-separation parameter on conjugation length, J. Chem. Phys., 2011, 135(20), 204107.

68 L. Kronik, T. Stein, S. Refaely-Abramson and R. Baer, Excitation Gaps of Finite-Sized Systems from Optimally Tuned Range-Separated Hybrid Functionals, J. Chem. Theory Comput., 2012, 8(5), 1515-1531. 
69 T. M. Wilson, M. J. Tauber and M. R. Wasielewski, Toward an $\mathrm{n}$-Type Molecular Wire: Electron Hopping within Linearly Linked Perylenediimide Oligomers, J. Am. Chem. Soc., 2009, 131(25), 8952-8957.

70 J. Gierschner, J. Cornil and H. J. Egelhaaf, Optical Bandgaps of $\pi$-Conjugated Organic Materials at the Polymer Limit: Experiment and Theory, Adv. Mater., 2007, 19(2), 173-191.

71 H. Sirringhaus, P. J. Brown, R. H. Friend, M. M. Nielsen, K. Bechgaard, B. M. W. Langeveld-Voss, A. J. H. Spiering, R. A. J. Janssen, E. W. Meijer, P. Herwig and D. M. de Leeuw, Two-dimensional charge transport in self-organized, highmobility conjugated polymers, Nature, 1999, 401(6754), 685-688.

72 R. Steyrleuthner, M. Schubert, I. Howard, B. Klaumünzer, K. Schilling, Z. Chen, P. Saalfrank, F. Laquai, A. Facchetti and D. Neher, Aggregation in a High-Mobility n-Type LowBandgap Copolymer with Implications on Semicrystalline Morphology, J. Am. Chem. Soc., 2012, 134(44), 18303-18317.

73 J. Clark, C. Silva, R. H. Friend and F. C. Spano, Role of Intermolecular Coupling in the Photophysics of Disordered Organic Semiconductors: Aggregate Emission in Regioregular Polythiophene, Phys. Rev. Lett., 2007, 98(20), 206406.

74 C. Scharsich, F. S. U. Fischer, K. Wilma, R. Hildner, S. Ludwigs and A. Köhler, Revealing structure formation in PCPDTBT by optical spectroscopy, J. Polym. Sci., Part B: Polym. Phys., 2015, 53(20), 1416-1430.

75 R. Brosi, B. Illarionov, T. Mathes, M. Fischer, M. Joshi, A. Bacher, P. Hegemann, R. Bittl, S. Weber and E. Schleicher, Hindered Rotation of a Cofactor Methyl Group as a Probe for Protein-Cofactor Interaction, J. Am. Chem. Soc., 2010, 132(26), 8935-8944.

76 C. Heller and H. M. McConnell, Radiation Damage in Organic Crystals. II. Electron Spin Resonance of
$\left(\mathrm{CO}_{2} \mathrm{H}\right) \mathrm{CH}_{2} \mathrm{CH}\left(\mathrm{CO}_{2} \mathrm{H}\right)$ in $\beta$-Succinic Acid, J. Chem. Phys., 1960, 32(5), 1535-1539.

77 D. M. Murphy and R. D. Farley, Principles and applications of ENDOR spectroscopy for structure determination in solution and disordered matrices, Chem. Soc. Rev., 2006, 35(3), 249-268.

78 B. P. Cherniawski, S. Lopez, E. K. Burnett, I. Yavuz, L. Zhang, S. Parkin, S. C. B. Mansfield, K. N. Houk and A. L. Briseno, in preparation.

79 S. Stoll and A. Schweiger, EasySpin, a comprehensive software package for spectral simulation and analysis in EPR, J. Magn. Reson., 2006, 178(1), 42-55.

80 W. B. Mims, Pulsed Endor Experiments, Proc. R. Soc. London, Ser. A, 1965, 283(1395), 452-457.

81 N. C. Miller, E. Cho, M. J. N. Junk, R. Gysel, C. Risko, D. Kim, S. Sweetnam, C. E. Miller, L. J. Richter, R. J. Kline, M. Heeney, I. McCulloch, A. Amassian, D. Acevedo-Feliz, C. Knox, M. R. Hansen, D. Dudenko, B. F. Chmelka, M. F. Toney, J.-L. Brédas and M. D. McGehee, Use of X-Ray Diffraction, Molecular Simulations, and Spectroscopy to Determine the Molecular Packing in a Polymer-Fullerene Bimolecular Crystal, Adv. Mater., 2012, 24(45), 6071-6079.

82 D. M. DeLongchamp, R. J. Kline, E. K. Lin, D. A. Fischer, L. J. Richter, L. A. Lucas, M. Heeney, I. McCulloch and J. E. Northrup, High Carrier Mobility Polythiophene Thin Films: Structure Determination by Experiment and Theory, Adv. Mater., 2007, 19(6), 833-837.

83 E. Cho, C. Risko, D. Kim, R. Gysel, N. Cates Miller, D. W. Breiby, M. D. McGehee, M. F. Toney, R. J. Kline and J.-L. Bredas, Three-Dimensional Packing Structure and Electronic Properties of Biaxially Oriented Poly(2,5-bis(3alkylthiophene-2-yl)thieno[3,2-b]thiophene) Films, J. Am. Chem. Soc., 2012, 134(14), 6177-6190. 